\title{
Dificultades en el Reconocimiento de Patrones Entonativos por Parte de Estudiantes de ELE
}

\author{
José Antonio Martín Gómez \\ Josefa DorTA LuIS \\ Universidad de La Laguna \\ Hirotaka Sensui \\ Nanzan University
}

Recibido: 01 mayo 2017 / Aceptado: 19 junio 2017

ISSN: $1697-7467$

RESUMEN: Se ha demostrado en estudios previos (Cortés Moreno, 2005; Sensui, 2015) que los estudiantes de español tienen dificultades con algunos patrones acentuales y entonativos, tanto en la producción como en la percepción, lo que causa frecuentemente identificaciones incorrectas de la modalidad de frase. Este trabajo tiene como objetivo estudiar este tipo de dificultades en estudiantes japoneses de ELE para sentar las bases de la creación de materiales específicos que atiendan al problema de reconocimiento incorrecto de la modalidad en el español y más concretamente en las variedades meridionales. Para asegurar que la modalidad se puede percibir solamente a través de rasgos prosódicos, hemos diseñado un test perceptivo de identificación con estímulos interrogativos y declarativos resintetizados sin contenido léxico-semántico obtenidos de emisiones naturales de dos variedades de español (septentrional, del norte de Madrid y atlántica, de las Islas Canarias). Establecemos dos hipótesis: la primera presupone que los jueces identificarán mejor la modalidad interrogativa septentrional dado que generalmente es la que se enseña como estándar; la segunda plantea que los resultados obtenidos permitirán encontrar los puntos de la prosodia más complicados de reconocer por parte de los alumnos japoneses, lo que permitirá mejorar la enseñanza de los distintos patrones principales del español y disminuir los errores de comprensión de los estudiantes.

Palabras clave: Percepción, identificación, estudiantes japoneses de ELE, prosodia, entonación.

Difficulties in the recognition of intonation patterns by ELE students

\begin{abstract}
Previous studies (Cortés Moreno, 2005; Sensui, 2015) show that students of Spanish language have difficulties with some intonation and stress patterns in terms of both production and perception, which frequently leads to the incorrect identification of the modality of sentences. The objective of the present work is to study this type of difficulties in Japanese students of ELE in order to help develop specific learning resources that take into account the problem of the incorrect identification of modalities in the Spanish language; particularly in its southern-most varieties. To ensure that modality is perceived exclusively through prosodic features, we have designed a perceptive identification test using re-synthetized interrogative and declarative stimuli without lexical-semantic content, which have been obtained from natural utterances of two varieties of the Spanish language (a northern variety from the north of Madrid, and an Atlantic variety from the Canary Islands). We put forward
\end{abstract}


two hypotheses: the first one establishes that judges will be able to identify the northern interrogative modality better, since this is generally taught as a standard; and the second one proposes that results obtained will allow identifying the prosodic features that are more difficult to recognise by Japanese students, which will help to improve the teaching of the main different patterns of the Spanish language, and hence diminish understanding errors.

Keywords: Perception, identification, Japanese students of ELE, prosody, intonation.

\section{INTRODUCCIÓN}

Las semejanzas en el nivel fónico entre el español y otras lenguas es uno de los motivos, según Sunden (2013:148-149), para propiciar que los estudiantes de ELE se interesen por el aprendizaje de la lengua. En el caso concreto del español y japonés, podemos ver semejanzas como, por ejemplo, poseer un sistema vocálico de cinco unidades o similitud en algunos fonemas consonánticos; además, es clara la preferencia por la estructura silábica del tipo $\mathrm{CV}$, aunque con muchas más restricciones en japonés. No obstante, tanto en el sistema vocálico como en el consonántico, existen algunas diferencias; por ejemplo, el redondeamiento de la [u] española no se da en la [u] japonesa, ni tampoco existe en japonés determinados contrastes fonológicos como, por ejemplo, el de la lateral /1/, la vibrante múltiple /r/ y la percusiva /r/. Fernández Lázaro et al. (2013), no obstante, consideran que es un falso mito la similitud fonético-fonológica entre las dos lenguas según reflejan algunos análisis concretos de errores de estudiantes japoneses por lo que manifiestan que hay que elaborar un material específico para tratar los problemas concretos del alumnado. Con todo, algunas encuestas (Ura, 2010:26, 33) indican que los estudiantes japoneses consideran que al estudiar español tendrán menos problemas en el aprendizaje de la pronunciación que en otros niveles lingüísticos, como la gramática.

En el plano prosódico, que es el que interesa en este trabajo, se ha visto que los estudiantes japoneses presentan algunos problemas. Así, por ejemplo, Hara (1990) señala la acentuación inadecuada en palabras átonas como mi, nuestro (antepuesto a un sustantivo), que, o se. Morimoto (2007:172-174), por su parte, se refiere a la dificultad derivada de la diferencia acentual entre el español (acento de intensidad) y el japonés (acento tonal) o/y de la estructura silábica. Desde el punto de vista investigador, Sensui (2015:78) señala la escasez de trabajos que tengan en cuenta el aprendizaje o la enseñanza de elementos prosódicos, como el acento o la entonación en contrapartida con los que tratan el estudio de otras parcelas lingüísticas, como la morfología o la sintaxis. Con todo, hay excepciones basadas, además, en alguna técnica experimental, que abordan el estudio de los elementos prosódicos, tanto desde el punto de vista de la producción como desde la percepción, algunas son las de Kimura et al. (2008), Sensui et al. (2008), Fernández Lázaro (2009), Kimura et al. (2010), Kimura et al. (2012), Takasawa et al. (2012), Sensui (2014), Sensui (2015), entre otras.

Entre los estudios prosódicos con base experimental, hay pocos sobre la producción del español por parte de estudiantes japoneses. Cabe citar al respecto a Sensui (2014: 24) quien analiza un corpus de oraciones emitidas por cuatro estudiantes japoneses de español. Este autor observa algunas tendencias en la producción de estos estudiantes como la anticipación del primer pico tonal, el movimiento ascendente retrasado en la parte final de las 
oraciones interrogativas, un registro tonal más compacto en estas mismas oraciones o un patrón ascendente-descendente en la concatenación de las palabras átonas.

En los estudios prosódicos de tipo perceptivo destacamos, por el objetivo del presente trabajo, algunos en los que se trata la modalidad, sobre todo declarativa e interrogativa. Kimura et al. (2008), Kimura et al. (2010), Sensui et al. (2008) concluyen que los estudiantes japoneses identifican el tipo de oración fundamentalmente por el movimiento tonal final. Fernández Lázaro (2009), por su parte, concluye que sus informantes japoneses presentan menos dificultades el percibir el patrón enunciativo que el exclamativo. Sensui (2015) confirma, con la aplicación de experimentos perceptivos, que los estudiantes japoneses de español discriminan la modalidad principalmente gracias a la parte final de la curva entonativa; indica, asimismo, que aunque son capaces de percibir con acierto los patrones declarativos (descendentes) e interrogativos (ascendentes), tienden a confundir el patrón interrogativo descendente, usado en algunas preguntas del español peninsular, con el declarativo debido a la caída del tono en la parte final que se da en ambos patrones.

\section{LA PROSODIA DEL JAPONÉS}

Desde el punto de vista tipológico el japonés es una lengua aglutinante, lo que implica que las partículas desempeñan un papel fundamental para marcar relaciones sintácticas o expresar la modalidad oracional. Véanse los ejemplos de la tabla 1:

Tabla 1. Oraciones declarativa e interrogativas, con y sin partícula, del japonés

\begin{tabular}{|c|c|c|c|c|c|c|c|}
\hline (1) & $\begin{array}{l}\text { Suzuki } \\
\text { Suzuki } \\
\text { [apellido] }\end{array}$ & $\begin{array}{l}\text { san } \\
\text { señor }\end{array}$ & $\begin{array}{l}\text { wa } \\
\text { partícula } \\
\text { temática }\end{array}$ & $\begin{array}{l}\text { koko } \\
\text { aquí }\end{array}$ & $\begin{array}{l}n i \\
\text { partícula } \\
\text { direccional }(a)\end{array}$ & $\begin{array}{l}\text { kimasu } \\
\text { viene }\end{array}$ & \\
\hline \multicolumn{8}{|c|}{ 'El señor Suzuki viene aquí' } \\
\hline (2) & $\begin{array}{l}\text { Suzuki } \\
\text { Suzuki } \\
\text { [apellido] }\end{array}$ & $\begin{array}{l}\text { san } \\
\text { señor }\end{array}$ & $\begin{array}{l}\text { wa } \\
\text { partícula } \\
\text { temática }\end{array}$ & $\begin{array}{l}\text { koko } \\
\text { aquí }\end{array}$ & $\begin{array}{l}n i \\
\text { partícula } \\
\text { direccional }(a)\end{array}$ & $\begin{array}{l}\text { kimasu } \\
\text { viene }\end{array}$ & $\begin{array}{l}\boldsymbol{k a} ? \\
\text { partícula } \\
\text { interrogativa }\end{array}$ \\
\hline \multicolumn{8}{|c|}{ “¿El señor Suzuki viene aquí?” } \\
\hline (3) & $\begin{array}{l}\text { Suzuki } \\
\text { Suzuki } \\
\text { [apellido] }\end{array}$ & $\begin{array}{l}\text { san } \\
\text { señor }\end{array}$ & $\begin{array}{l}\text { wa } \\
\text { partícula } \\
\text { temática }\end{array}$ & $\begin{array}{l}\text { koko } \\
\text { aquí }\end{array}$ & $\begin{array}{l}n i \\
\text { partícula } \\
\text { direccional }(a)\end{array}$ & $\begin{array}{l}\text { kimasu? } \\
\text { viene }\end{array}$ & - \\
\hline
\end{tabular}


De los ejemplos de la tabla 1, (1) se interpreta como enunciativa al carecer de la partícula $k a$ que vemos en (2) y, por ello, se emite con un movimiento tonal descendente; (2), en cambio, se interpreta como interrogativa puesto que ka es marca de pregunta y la oración se pronuncia normalmente con entonación ascendente. Con todo, como se ve en (3), en el estilo coloquial o informal se interpreta una oración como interrogativa si se pronuncia con un movimiento tonal ascendente en la parte final aun careciendo de la partícula interrogativa. Al respecto, Kori (2015) analiza los patrones entonativos finales sin partícula interrogativa y observa que el ascenso no siempre garantiza la interrogación ya que puede tener otros significados pragmáticos, como el énfasis. No obstante, las oraciones interrogativas (sobre todo absolutas) suelen pronunciarse con el patrón ascendente y de ahí que los hablantes japoneses sean capaces de asociar la entonación ascendente con la modalidad interrogativa en español.

\section{Patrones entonativos y objetivo}

Como se ha visto en el apartado anterior, los japoneses identifican la entonación final ascendente del español con la modalidad interrogativa puesto que usan un patrón ascendente $/ \mathrm{H} \% /$ en su lengua materna para la misma finalidad. Ahora bien, en español coexisten varios patrones nucleares para marcar dicha modalidad: el patrón $/ \mathrm{H} \% /$ es el más usado en el español estándar peninsular, mientras que el patrón ascendente-descendente o circunflejo es el más común en muchos países hispanohablantes por lo que es usado por muchos millones de personas. Véase un esquema de ambos en la figura 1.

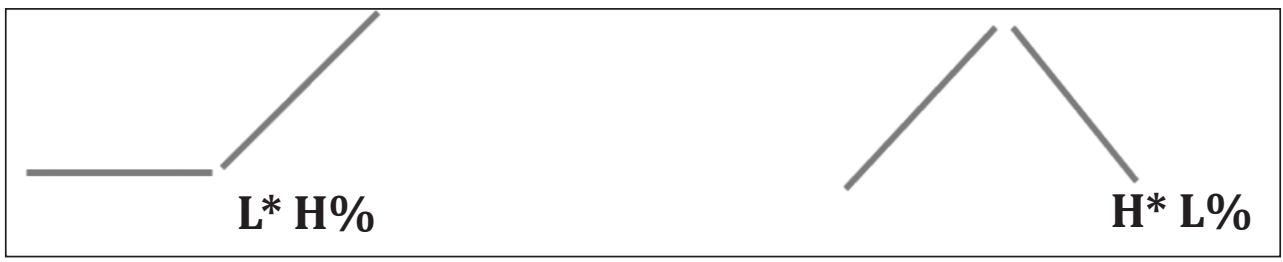

Figura 1. Esquemas interrogativos nucleares ascendente y circunflejo

En efecto, en estudios previos (Dorta, 2013; Dorta, Martín y Díaz, 2015, entre otros) se ha probado que las interrogativas de Canarias, Cuba, Venezuela o Colombia muestran un tono $/ \mathrm{H}^{*} /$ en la última sílaba acentuada y un final $/ \mathrm{L} \% /$, mientras que en las variedades peninsulares lo más común es un tono $/ \mathrm{L}^{*} /$ seguido de un final $/ \mathrm{H} \% /$ en la mayoría de interrogativas absolutas. La figura 2 ilustra la curva de f0 de una interrogativa con final ascendente de Madrid y las figuras 3 y 4 dos curvas superpuestas de declarativa (inferior) e interrogativa (superior) de Tenerife y de La Habana, respectivamente. En estas dos figuras se puede ver que las declarativas se diferencian de las interrogativas por el tono $/ \mathrm{H}^{*} /$ de la última tónica, ya que a partir del pico tonal nuclear la configuración tonal es descendente en las dos modalidades situándose el final absoluto prácticamente en la misma altura tonal. 


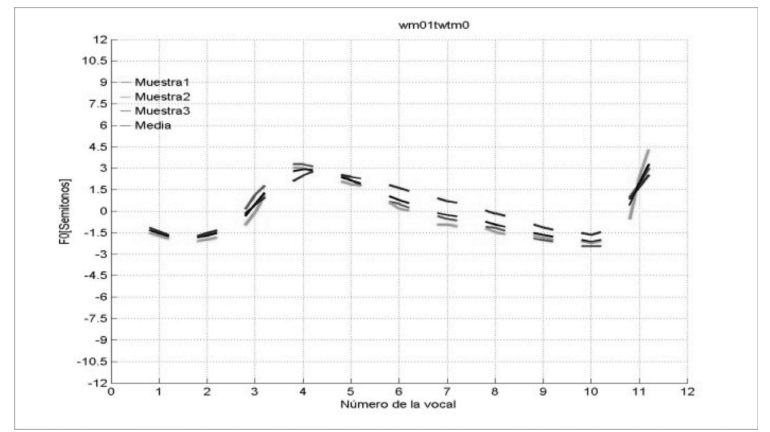

Figura 2. Media de interrogativas de una mujer de Madrid ${ }^{l}$
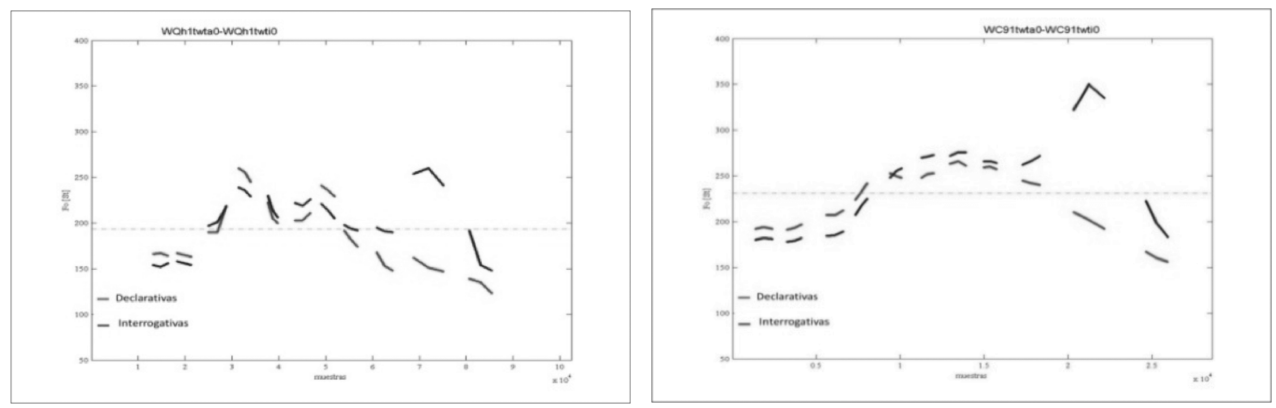

Figuras 3 y 4. Interrogativa y declarativa de una mujer de Tenerife (izquierda) y de La Habana (derecha)

Teniendo en cuenta la inexistencia de un patrón interrogativo único en español, nuestro objetivo prioritario es comprobar si los estudiantes japoneses de español pueden distinguir adecuadamente los patrones interrogativos mencionados, esto es, el ascendente de la variedad septentrional y el descendente (o circunflejo) de las variedades mencionadas. En el caso de distinguir adecuadamente solo el primero, ello implicaría confusiones muy importantes y un obstáculo en la comunicación con los millones de hablantes de las variedades que utilizan el segundo. Además de este objetivo fundamental, nos interesa observar si la modalidad interrogativa queda diferenciada de la declarativa. Para la consecución de nuestro objetivo, hemos realizado un test perceptivo a estudiantes japoneses de español con oraciones declarativas e interrogativas neutras del español peninsular, considerado como el estándar, y del español de Tenerife, cuya modalidad interrogativa muestra siempre, como se ha dicho, un final ascendente-descendente o circunflejo. En ambos casos consideramos los tres tipos acentuales del español -agudo, llano y esdrújulo- en el núcleo de las frases, ya que influyen considerablemente en la configuración tonal de la última parte de la oración (por ejemplo, el acento nuclear agudo produce un truncamiento del patrón interrogativo descendente de Canarias, como se explicará más adelante).

\footnotetext{
${ }^{1}$ Figura tomada de Martínez Celdrán y Fernández Planas (2003-2015).
} 


\section{Cuestiones metodológicas}

\subsection{El corpus y el test de percepción}

Para asegurar que la modalidad solo se percibe a través de rasgos prosódicos hemos preparado un test de identificación con estímulos resintetizados sin contenido léxico-semántico ${ }^{2}$ obtenidos de frases naturales (también añadidas al test) emitidas por mujeres de dos variedades del español, la madrileña y la canaria, caracterizadas por el final ascendente y descendente de sus interrogativas, respectivamente. En el corpus hemos alternado las palabras del núcleo de las frases para incluir los tres tipos acentuales del español (agudo, llano y esdrújulo), mientras que el verbo y el sujeto mantienen siempre acento llano (La guitarra se toca con paciencia/ con obsesión/ con pánico $)^{3}$. En definitiva, este corpus español consta de 48 estímulos sintetizados y frases naturales mezcladas; en el mismo se consideran las dos variedades mencionadas, los tres tipos de acento final y las modalidades declarativa e interrogativa.

Además del corpus español, se ha preparado también un corpus resintetizado del japonés estándar de Tokio con las mismas características que el corpus español ${ }^{4}$. El objetivo es comprobar si los japoneses son capaces de distinguir la modalidad de sus propias oraciones solo mediante la prosodia y sin la ayuda de la partícula interrogativa de que se ha hablado anteriormente e intentar inferir qué técnicas pueden usar para hacerlo o qué dificultades pueden tener. Así, hemos tratado de respetar la configuración silábica y acentual del corpus español para comprobar si el tono de la última palabra afecta o no a la percepción de la modalidad también en su lengua nativa, sobre todo al no tener pistas léxicas, como la partícula interrogativa. Véase en la tabla 2 el cómputo del conjunto de estímulos utilizados en los diferentes corpus y en la tabla 3 el diseño del corpus japonés.

\section{Tabla 2. Corpus de análisis}

\begin{tabular}{|c|c|c|c|c|c|c|}
\hline Corpus & Tipo de estímulo & Variedades & $\begin{array}{c}\text { Tipos de } \\
\text { acento }\end{array}$ & Modalidad & Repetición & Total \\
\hline Japonés & 1 & 1 & 3 & 2 & 2 & 12 \\
\hline Español & 2 & 2 & 3 & 2 & 2 & 48 \\
\hline
\end{tabular}

\footnotetext{
${ }^{2}$ Estos estímulos permiten oír el tono de cada vocal nuclear con un efecto parecido a una trompeta, por lo que no se puede averiguar otra información que no sea la prosódica, esto es, duración, intensidad y f0 de cada vocal.

${ }^{3}$ El corpus se ha elaborado siguiendo las directrices del proyecto AMPER (Atlas Multimedia de Prosodia del Espacio Románico). Este proyecto nace a finales del S. XX en Francia gracias a una idea de Michel Contini, investigador del Centro de Dialectología de la Universidad Stendhal-Grenoble III.

${ }^{4}$ La única diferencia es que del español se pasaron estímulos resintetizados y naturales, mientras que del japonés solo se incluyeron en el test los primeros para que los oyentes no supieran que se trataba de su propia lengua.
} 
Tabla 3. Corpus del test japonés

\begin{tabular}{|c|c|c|c|c|c|c|}
\cline { 5 - 7 } & \multicolumn{2}{l|}{ Aguda } & Llana & Esdrújula \\
\hline Ashita & taberu & no & wa & unagi & tamago & tomato \\
\hline$[\mathrm{LHH}]$ & HHL & $\mathrm{L}$ & $\mathrm{L}]$ & {$[\mathrm{LHH}]^{5}$} & {$[\mathrm{LHL}]$} & {$[\mathrm{HLL}]$} \\
\hline mañana & comer & partícula posesiva & partícula temática & anguila & huevo & tomate \\
\hline
\end{tabular}

Todas las frases han sido diseñadas para que tengan 11 vocales, 11 moras y 11 sílabas, algo que no es sencillo, dado que, como el japonés es una lengua moráica, en ocasiones puede que no coincida el número de estos tres elementos. Según Kubozono (1999:195-197), palabras como to-o-kyo-o (Tokyo) o ka-n-sa-i (Kansai, parte oeste de Japón) se dividen en cuatro elementos; por lo tanto, desde este punto de vista, las dos palabras (Tokyo y Kansai) tienen dos sílabas (onsetsu) y cuatro moras. Por ello hemos preparado el corpus del japonés de modo que no se produzcan conflictos de este tipo.

El test elaborado a partir de los corpus japonés y español se pasó dos veces de manera aleatoria a los jueces (estudiantes japoneses), con el fin de comprobar la coherencia de sus respuestas, y en orden aleatorio. En total cada participante escuchaba 60 estímulos.

\subsection{Auditores}

El test de identificación se pasó in situ a 60 auditores japoneses, estudiantes de español de las universidades Sophia y Seisen, en Tokio y Nanzan, en Nagoya. Todos cursaban tercero o cuarto curso del Grado en Español, Lengua y Cultura y contaban con un nivel B1 de español, por lo que eran capaces de expresarse y entender el español con solvencia. Como grupo de control seleccionamos un total de 14 japoneses sin conocimiento de español, a los que se pasó el test en las mismas condiciones que a los estudiantes de español con el fin de conocer si cometen más errores que estos, lo que querría decir que la instrucción de los estudiantes de español da ventaja en la tarea de identificación. En cambio, si los porcentajes de acierto de ambos grupos es similar o el mismo, se entendería que el porcentaje de error registrado tiene que ver más con la configuración de la lengua materna y evidenciaría una falta de preparación específica para los estudiantes de ELE.

\subsection{Programa TP}

Para realizar el test de identificación hemos usado el programa TP Test, versión 3.1 de Worken, una herramienta para este tipo de estudios de percepción. La pregunta que se le hizo a los participantes fue: ¿Esta frase es declarativa o interrogativa?; el auditor debía escoger una de las dos opciones, con la posibilidad de escuchar el estímulo hasta 4 veces en el caso de no estar seguro de su respuesta. El test se realizó con ordenadores y auriculares Sennheiser HD 407.

\footnotetext{
${ }^{5}$ Se ha elegido el tono [LHH] dado que no existe [LLH] en la variedad estándar; esa configuración tonal es la más parecida a la de una aguda del español.
} 


\section{Resultados obtenidos}

\subsection{Auditores estudiantes de ELE}

La tabla 4 muestra el porcentaje de error medio de los jueces estudiantes de ELE. Dado que se encontró una gran coherencia interjueces ${ }^{6}$, en ella se separan los resultados según la modalidad (declarativa vs. interrogativa), el tipo acentual final (agudo, llano y esdrújulo), el tipo de estímulo (natural o resintetizado) y la variedad de la informante (japonés de Tokio y español de Madrid y Tenerife).

Respecto de los estímulos resintetizados del japonés (como se dijo ya, no se pasaron las oraciones naturales de esta lengua), llama la atención el alto porcentaje de error en los declarativos con final agudo. Ello podría deberse a que, como mencionábamos en el apartado dedicado al corpus, la palabra unagi tiene una secuencia tonal [LHH] que puede motivar que los japoneses se confundan en la mitad de los casos con el patrón [H\%] de las interrogativas ya que, debido a las características del test, no tienen la oportunidad de escuchar la partícula interrogativa ni otro contexto que les ayude a resolver la ambigüedad presentada. En el resto de finales, se registran pocos errores, tanto en los estímulos declarativos como en interrogativos destacando particularmente el bajo porcentaje de error en los interrogativos con final llano. Hay que mencionar que en los declarativos hay más errores en los estímulos sintetizados de la propia lengua que en los del español, particularmente en los del español de Madrid, variedad esta que reconocen mejor. Ello implica que, o bien las diferencias prosódicas entre el español y el japonés les han confundido ${ }^{7}$, puesto que esperaban oír frases en español, o que en japonés no es suficiente la entonación para inferir la modalidad oracional en todos los casos (de ahí el uso de la partícula léxica ${ }^{8}$.

Al escuchar las oraciones naturales del español de Madrid, los estudiantes de ELE japoneses no muestran ninguna dificultad para señalar correctamente la modalidad oracional, pues los porcentajes de error llegan a ser incluso $0 \%$ en una muestra de 60 participantes. Ello demuestra que están muy acostumbrados a identificar ambas modalidades en español estándar. En cambio, sí se registran confusiones en las interrogativas resintetizadas ${ }^{9}$, sobre todo en el final llano $(28,33 \%)$, aunque los porcentajes de error no son muy elevados y podrían deberse al impacto de la falta de información segmental, dado que las oraciones naturales y filtradas presentadas son exactamente las mismas. Además, cabría esperar en este tipo de

\footnotetext{
${ }^{6}$ Como se dijo ya, cada estímulo era pasado dos veces a cada informante de manera aleatoria; al respecto, también se ha comprobado que existe una alta coherencia interinformante, dado que mayoritariamente respondían lo mismo en ambas ocasiones.

${ }^{7}$ En español el acento no es solamente tonal, por lo que interfiere mucho menos en la percepción de la curva entonativa de la frase.

${ }^{8}$ Hemos apuntado en la introducción que en el japonés coloquial la partícula puede evitarse; sin embargo en la comunicación oral hay varias pistas que ayudan a inferir la modalidad, por lo que parece que el tono de frontera final $\mathrm{H} \%$ no se revela como $100 \%$ relevante ya que además se confunde con el último tono de la palabra unagi.

${ }^{9}$ El rango de error en declarativas no naturales es algo mayor que en las naturales; sin embargo queda muy lejos incluso del porcentaje de error en su propia lengua materna.
} 
experimentos que, en caso de duda o de creer que no se tiene la suficiente información, los jueces tiendan a escoger la respuesta menos marcada, en este caso la modalidad declarativa.

Tabla 4. Resultados en porcentajes de respuesta incorrecta de los estudiantes japoneses de español para los diferentes corpus

\begin{tabular}{|c|c|c|c|c|c|c|c|}
\cline { 3 - 8 } \multicolumn{2}{c|}{} & \multicolumn{3}{c|}{ Declarativas } & \multicolumn{3}{c|}{ Interrogativas } \\
\cline { 2 - 8 } \multicolumn{2}{c|}{} & agudo & 1 llano & esdrújulo & agudo & llano & esdrújulo \\
\hline \multirow{3}{*}{ Japonés } & Natural & - & - & - & - & - & - \\
\cline { 2 - 8 } & Resint. & 52,50 & 14,17 & 15,00 & 10,83 & 5,83 & 15,83 \\
\hline \multirow{3}{*}{ Tadrid } & Natural & 0,00 & 2,50 & 0,83 & 4,17 & 0,00 & 2,50 \\
\cline { 2 - 8 } & Resint. & 6,67 & 6,67 & 5,83 & 16,67 & 28,33 & 17,50 \\
\hline & Natural & 3,33 & 1,67 & 3,33 & 7,50 & 66,67 & 79,17 \\
\cline { 2 - 8 } & Resint. & 21,67 & 4,17 & 5,00 & 14,17 & 41,67 & 82,50 \\
\hline
\end{tabular}

Por último, en los estímulos de Tenerife, los jueces cometen muy pocos errores en las declarativas naturales y en las sintetizadas aciertan incluso más que en las de su propia lengua, excepto en el acento final agudo (que en español se marca con una combinación de intensidad, f0 y duración) donde se interpretan como interrogativas en algo más del $20 \%$ de las ocasiones. En las interrogativas, en cambio, el porcentaje de errores solo se mantiene bajo en los finales agudos, tanto naturales como sintetizados. Ello se debe a que, como se muestra en la figura 5, las interrogativas agudas del patrón circunflejo suelen terminar con ascenso de la f0 debido a que muchas veces se da un "truncamiento tonal" que no deja observar el patrón tonal circunflejo subyacente ${ }^{10}$. En efecto, como se ha documentado en diversos trabajos (v. gr. Dorta, 2008; Dorta, 2013 o Dorta y Martín, 2014), la característica principal del patrón circunflejo es un tono $/ \mathrm{H}^{*} /$ en la última tónica que contrasta con el tono $/ \mathrm{L}^{*} /$ del núcleo de las declarativas. El tono de frontera final en ambas modalidades es /L\%/ pero, en el caso de las interrogativas con final agudo, al truncarse la f0, la regla del patrón circunflejo queda esbozada de la manera siguiente:

La tónica debe ser $\mathrm{H}$ y el final L. La primera parte es la única inviolable y la segunda se cumple siempre que haya suficiente espacio tras la tónica. Por tanto, de acuerdo con esta regla, el final agudo ascendente esconde un patrón fonológico circunflejo puesto que no viola la regla: muestra un tono $\mathrm{H}$ en la tónica y si no

\footnotetext{
${ }^{10}$ Esta misma tendencia se encuentra en interrogativas con final agudo de Cuba, Venezuela y Colombia.
} 
finaliza en $\mathrm{L}$ es porque no existen sílabas después de la tónica que le permitan terminar con un tono bajo. En definitiva, no parece coherente que hablemos de dos patrones fonológicos por lo que proponemos que, en el caso de los finales agudos, se hable de "patrón circunflejo truncado" o "patrón circunflejo con final ascendente", aclarando siempre que este patrón es el mismo que el que se da en llanos y esdrújulos (Dorta y Martín, 2014: 200).

Lógicamente, los estudiantes de ELE encuestados confunden el "patrón circunflejo truncado" o "patrón circunflejo con final ascendente" de los estímulos interrogativos agudos, con un patrón $/ \mathrm{H} \% /$ y por ello aciertan identificando la modalidad en la mayoría de los casos. No obstante, y como se aprecia en la figura 6, las interrogativas con final llano y esdrújulo sí presentan un final descendente, que la mayoría de las veces culmina a la par de los finales declarativos, por lo que el número de respuestas incorrectas se dispara, tanto en las oraciones naturales como en las sintéticas. En este caso interpretamos que los jueces entienden esta subida del tono en la tónica como una marca de acento (bien sea llano o esdrújulo) y se fían exclusivamente del final descendente para reconocer la modalidad. En las interrogativas con final esdrújulo hay dos sílabas entre la tónica $/ \mathrm{H}^{*} /$ y el final, por lo que no sólo el pico queda más lejos del final para los jueces, sino que hay más tiempo para que la curva descienda en mayor medida ${ }^{11}$, lo que provoca resultados de confusión mayores que en los finales llanos. Si comparamos los resultados obtenidos con nuestra primera hipótesis, se cumple sin ninguna duda que los estudiantes japoneses de ELE identifican mejor la modalidad interrogativa septentrional (y también la declarativa en los casos de resíntesis) dado que es la variedad más enseñada al considerarse estándar. También se aprecia en este apartado cómo los resultados indican que la modalidad interrogativa es la que más confusión genera en los estudiantes, sobre todo debido a que los alumnos se fijan exclusivamente en el movimiento final de la oración donde, además, se mezcla el movimiento entonativo de la f0, indicador de la modalidad, con la marca de cada tipo acentual, una combinación de duración, f0 e intensidad. Estos resultados permiten, como planteábamos en nuestra segunda hipótesis, conocer el punto al que los estudiantes deben prestar más atención para dominar los patrones principales del español.

\footnotetext{
${ }^{11}$ Las interrogativas con final esdrújulo suelen registrar, según nuestros estudios, fuertes descensos que culminan incluso por debajo de sus homólogas declarativas.
} 

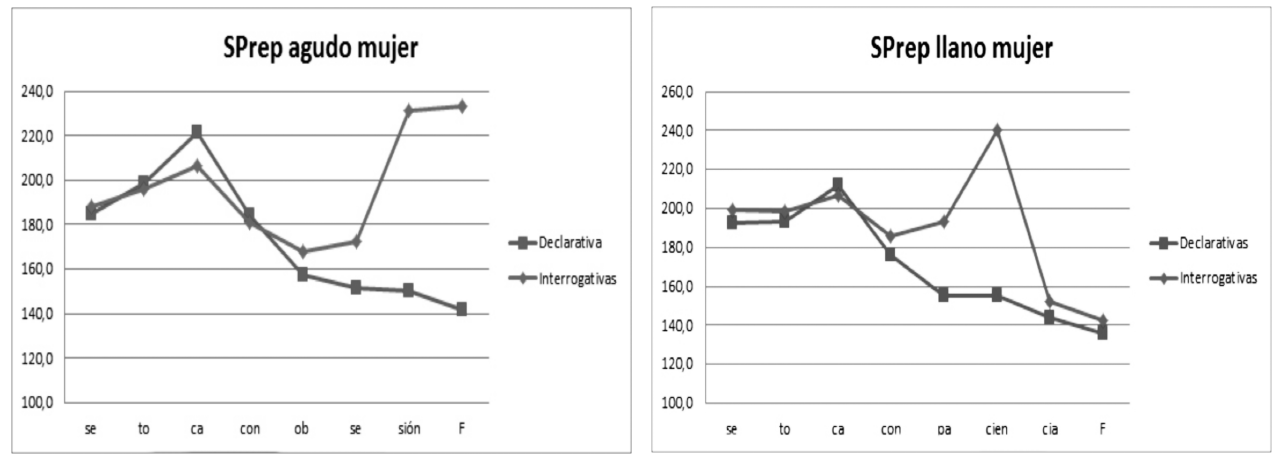

Figuras 5 (izquierda) y 6 (derecha). Declarativas e interrogativas tinerfeñas con final agudo y llano

\subsection{Auditores sin conocimientos de ELE}

Como se ha dicho ya, realizamos el mismo test a 14 nativos japoneses como grupo de control sin conocimiento alguno de español con la finalidad de encontrar si el entrenamiento de los estudiantes de ELE en la pronunciación del español les hace identificar mejor la modalidad oracional, al menos en alguna de las variedades, respecto de los que carecen de formación y nunca han conversado en español.

En la tabla 5 observamos que la cantidad de errores en el reconocimiento de la modalidad en el corpus sintetizado del japonés es similar, e incluso superior, a la de los estudiantes de ELE, lo que hace pensar que sin la información léxica y, en el caso de las interrogativas, sobre todo sin la partícula $k a$, es difícil para los japoneses identificar la modalidad con total seguridad. Hay que destacar, nuevamente, que en los estímulos declarativos (como ocurría en la tabla 4 para los estudiantes de ELE) se evidencia la confusión en más de la mitad de casos de la declarativa cuya última palabra registra los tonos léxicos [LHH] con una interrogativa con final $[\mathrm{H} \%]$.

Tabla 5. Resultados de porcentaje de respuesta incorrecta de los japoneses sin conocimientos de español para los diferentes corpus

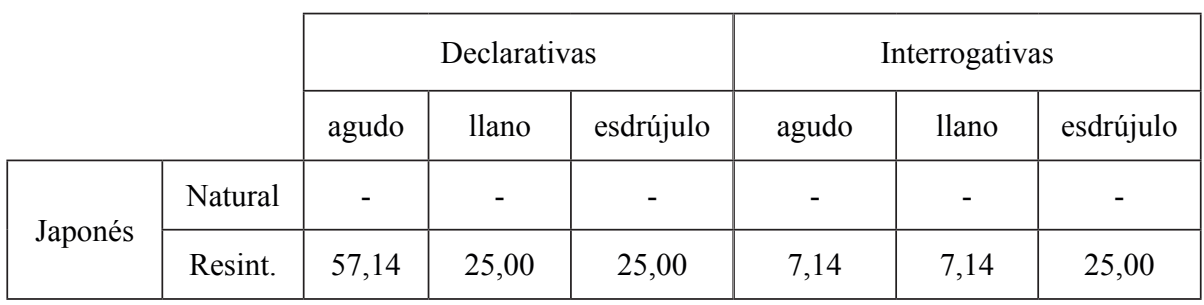




\begin{tabular}{|c|c|c|c|c|c|c|c|}
\hline \multirow{2}{*}{ Madrid } & Natural & 10,71 & 14,29 & 7,14 & 3,57 & 0,00 & 0,00 \\
\cline { 2 - 8 } & Resint. & 32,14 & 14,29 & 7,14 & 21,43 & 39,29 & 0,00 \\
\hline \multirow{2}{*}{ Tenerife } & Natural & 14,29 & 0,00 & 25,00 & 0,00 & 78,57 & 50,00 \\
\cline { 2 - 8 } & Resint. & 17,86 & 0,00 & 10,71 & 35,71 & 46,43 & 85,71 \\
\hline
\end{tabular}

Por otra parte, los japoneses se muestran mucho más competentes al identificar las declarativas e interrogativas naturales de Madrid (sobre todo estas últimas) independientemente del acento final, con resultados similares, aunque no superiores, a los de los estudiantes de español ${ }^{12}$. Quizás ello se deba a que, como hemos dicho, en español el acento se marca con una combinación de parámetros que modifica menos la curva entonativa de la frase. En cambio, en los estímulos sintetizados registran porcentajes de error superiores (sobre todo en agudos de las declarativas y en llanos de las interrogativas) respecto de los estudiantes de ELE. Se exceptúan los estímulos interrogativos con final esdrújulo pues no registran errores, al contrario de lo que sucede en los estudiantes de ELE aunque el porcentaje de errores de estos últimos no sea muy alto (17,5\%). Teniendo en cuenta el reconocimiento de los estímulos japoneses y el de los españoles de Madrid, no queda suficientemente probado que el posible entrenamiento de los estudiantes de ELE en los patrones entonativos del español estándar sea suficientemente bueno puesto que sus resultados no se distancian en gran medida de los obtenidos de los japoneses que no saben español.

Respecto de las oraciones de Tenerife observamos que en las declarativas los porcentajes de error más llamativos respecto de los obtenidos por los estudiantes de ELE son los de las oraciones naturales con final agudo y esdrújulo y, fundamentalmente, que en los llanos no cometen errores ni en los estímulos naturales ni en los sintetizados (aunque en este caso los porcentajes de error de los estudiantes de ELE son muy bajos). En las interrogativas, tanto naturales como sintetizadas, la diferencia más importante entre los dos grupos de auditores se da en los agudos, sobre todo en los estímulos sintéticos pues en estos los japoneses no conocedores del español tienen más del doble de errores que los estudiantes de ELE. Por otra parte, en aquellos se observa cierta incongruencia en el reconocimiento de las agudas puesto que identifican perfectamente como interrogativas las naturales, pero fallan un $36 \%$ en los estímulos sintetizados (en este caso se encuentra mayor coherencia en los estudiantes de ELE). En cambio, en las interrogativas con núcleo llano y esdrújulo encontramos resultados muy parecidos en los dos grupos ${ }^{13}$, puesto que los errores se disparan en estos dos tipos acentuales, lo que parece demostrar que los estudiantes de ELE no tienen ningún tipo de entrenamiento para reconocer el patrón circunflejo, por lo que lo hacen igual de mal que los japoneses sin conocimiento de español que usan los esquemas de su lengua materna

\footnotetext{
${ }^{12}$ En las esdrújulas de interrogativas registran $0 \%$ de errores, pero los estudiantes de ELE tienen solamente 2,50\% y se trata de un grupo mucho mayor.

${ }^{13}$ Como se ha dicho, el grupo de control de los japoneses no está integrado por demasiados informantes por lo que no se debe tener en cuenta el porcentaje exacto de error en la comparación con el grupo de ELE pero sí la tendencia, que en este caso es muy clara.
} 
para intentar identificar la modalidad (la técnica mencionada por otros autores de atender solamente al tono del final absoluto).

En las tablas 6 y 7 mostramos el total de estímulos analizados y el porcentaje de error para cada variedad independientemente de la modalidad, el tipo de oración o del acento final. Como se puede observar, los jueces del grupo de control tienen casi el doble de errores en los estímulos de la variedad de Madrid respecto de los estudiantes de ELE, pero ambos grupos se aproximan en porcentaje de error en los estímulos de Tenerife y de su lengua materna.

Tabla 6. Porcentaje de error total de estudiantes japoneses de ELE según la variedad

\begin{tabular}{|c|c|c|c|c|}
\hline \multicolumn{2}{|c|}{ Total estímulos por variedad } & Aciertos & Errores & Porcentaje de errores \\
\hline Japonés & 720 & 583 & 137 & 19,03 \\
\hline Madrid & 1440 & 1330 & 110 & 7,64 \\
\hline Tenerife & 1440 & 1043 & 397 & 27,57 \\
\hline
\end{tabular}

Tabla 7. Porcentaje de error total de japoneses sin conocimiento de español según la variedad

\begin{tabular}{|c|c|c|c|c|}
\hline \multicolumn{2}{|l|}{ Total errores por variedad } & Aciertos & Errores & $\begin{array}{c}\text { Porcentaje de } \\
\text { errores }\end{array}$ \\
\hline Japonés & 168 & 127 & 41 & 24,40 \\
\hline Madrid & 336 & 294 & 42 & 12,50 \\
\hline Tenerife & 336 & 229 & 107 & 31,85 \\
\hline
\end{tabular}

Con los resultados expuestos anteriormente para el grupo de estudiantes de español hay que deducir que si un japonés hablante de español como LE es preguntado por un hablante canario o hispanoamericano que use el patrón $/ \mathrm{H}^{*} \mathrm{~L} \% /$ (circunflejo), independientemente del tipo acentual de la última sílaba, tiene una gran posibilidad de equivocarse al intentar identificar la modalidad de la frase. La figura 7 muestra esa posibilidad de confusión; para calcular los porcentajes de la misma hemos reunido todos los resultados de las preguntas naturales de Tenerife (tabla 8). Además, este porcentaje de error podría ser mayor en una situación comunicativa real teniendo en cuenta la ocurrencia de los tipos acentuales del español, donde el llano, que es mayoritario, confundía a los japoneses en un $67 \%$ según nuestros resultados (tabla 4). 


\section{Probabilidad de error al oír una pregunta de tipo $\mathrm{H}^{*} \mathrm{~L} \%$}

\section{Aciertos Errores}

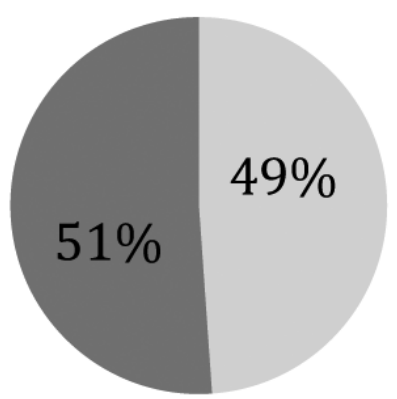

Figura 7. Probabilidad de error al oír una pregunta de tipo $H^{*} L \%$

Tabla 8. Determinación del porcentaje de error en la percepción de estímulos con patrón $/ H^{*} L \% /$

\begin{tabular}{|c|c|c|c|}
\hline Total estímulos interrogativos & Aciertos & Errores & $\%$ errores \\
\hline $360^{14}$ & 176 & 184 & $51,11 \%$ \\
\hline
\end{tabular}

\section{Discusión}

Fernández Lázaro et al. (2013) etiquetan de "falso mito" la similitud fonético-fonológica entre el español y el japonés. Tal consideración podemos hacerla extensiva al ámbito prosódico al que hemos atendido en este trabajo puesto que el concepto de acento en español, con sus tres tipos acentuales implicados en nuestro estudio, difiere bastante del japonés por lo que la diferencia puede motivar errores en la interpretación de los patrones tonales. Al respecto,

\footnotetext{
${ }^{14}$ Esta cifra es el resultado de 3 estímulos naturales más 3 resintetizados multiplicado por 60 estudiantes.
} 
Morimoto (2007:172-174) habla de la dificultad que puede tener el hecho de que el español presente acento de intensidad ${ }^{15}$ y el japonés acento tonal. Sensui (2015:78) señala, por su parte, que son pocos los trabajos que tienen como objetivo la descripción de las dificultades en el aprendizaje de la prosodia del español. El presente trabajo constituye una aportación en ese sentido al considerar, además de la variedad estándar frecuente en estudios de tipo perceptivo, la percepción de la prosodia de la variedad meridional. El mismo autor (Sensui 2014: 24) comprueba la tendencia de los estudiantes a pronunciar las oraciones interrogativas con un patrón final ascendente por la interferencia de su L1, algo que también afecta a su percepción según nuestros resultados ya que esperan que la marca de modalidad interrogativa sea un tono $/ \mathrm{H} \% /$. Pruebas de ello son el alto grado de acierto en las interrogativas agudas de Tenerife con final ascendente y la confusión de las interrogativas resintetizadas de Madrid con final ascendente con una declarativa si el tono de frontera no sube demasiado. Por último, Sensui (2015) indica que los estudiantes japoneses no muestran grandes dificultades para percibir con acierto los patrones declarativos (descendentes) e interrogativos (ascendentes) pero que tienden a confundir el patrón interrogativo descendente, que se encuentra en algunas preguntas del español peninsular, con el declarativo. Al respecto, en este trabajo se confirma que no solo confunden el patrón descendente de algunas interrogativas del español estándar, sino que tienen problemas para reconocer las interrogativas neutras tinerfeñas que, por tener un final circunflejo, se diferencian de las interrogativas del estándar y en cambio se acercan a las de muchas variedades meridionales como el español de Cuba o Venezuela (Dorta, 2013; Fernández Planas et al., 2015).

\section{Conclusiones}

$1^{\text {a) }}$ Las oraciones declarativas del español, similares en la variedad septentrional y la canaria, son identificadas correctamente con gran precisión por los estudiantes japoneses de ELE; solo en las oraciones resintetizadas con final agudo de Tenerife se da un porcentaje de error algo superior al $20 \%$. En los japoneses sin conocimientos de español, en general, la tasa de aciertos en esta modalidad es algo menor que en los estudiantes de español. No obstante, el margen diferencial entre ambos grupos no evidencia que la formación específica de los estudiantes de ELE haya influido en un mejor reconocimiento. Esta conclusión se refuerza si tenemos en cuenta que ambos grupos de auditores confunden, incluso más, los estímulos sintetizados de su propia lengua, especialmente los finales agudos de las declarativas con las interrogativas de tipo ascendente.

$\left.2^{a}\right)$ Respecto a las interrogativas peninsulares con final ascendente $/ \mathrm{L} * \mathrm{H} \% /$, se ha comprobado que los dos grupos de japoneses reconocen excepcionalmente bien las interrogativas naturales; las resintetizadas, en cambio, provocan un mayor porcentaje de error, con una cifra ligeramente inferior en los estudiantes de español que en

\footnotetext{
${ }^{15}$ Que la intensidad sea el parámetro fundamental del acento español no es, precisamente, la opinión más extendida; no obstante, el objetivo de la discusión en este momento no es la naturaleza de este prosodema.
} 
el otro grupo sin experiencia del idioma. Este último, en cambio, curiosamente no presenta error alguno en los esdrújulos. Con todo, la media de error en esta modalidad se sitúa en el $11,1 \%$ y en el $20,5 \%$ si solo tenemos en cuenta los errores en los estímulos modificados.

$3^{a}$ ) Al contrario de lo que sucede en las interrogativas del español septentrional, los dos grupos fallan estrepitosamente al identificar las preguntas con patrón circunflejo $/ \mathrm{H}^{*}$ L\%/, lo que indica que los estudiantes de ELE están usando las mismas herramientas que los que no saben español a la hora de seguir las pistas acústicas para distinguir la modalidad, como adscribir el tono $/ \mathrm{H}^{*} /$ de la última tónica a un acento de palabra, en lugar de tonal, y fiarse solamente del tramo final para reconocer la modalidad oracional (esperando además un tono ascendente para la interrogativa). El uso de estas tácticas se debe muy probablemente a la influencia de su lengua materna y del inglés (usado habitualmente como lengua vehicular y de la cual el japonés toma gran cantidad de elementos). También pueden haberse reforzado en las clases de ELE, que quizás hacen demasiado énfasis en la forma ascendente de las oraciones interrogativas del español estándar sin mencionar prácticamente otros patrones. Ello determina el reconocimiento de manera aceptable del patrón interrogativo ascendente, como hemos comprobado, pero el conocimiento de los alumnos no es adecuado para identificar el circunflejo. De ahí, precisamente, que ambos grupos registren una buena tasa de aciertos en las interrogativas de Tenerife con final agudo puesto que, debido a su truncamiento tonal, terminan en un tono alto.

$4^{\text {a }) ~ L a s ~ o r a c i o n e s ~ s i n t e t i z a d a s ~ d e l ~ j a p o n e ́ s ~ p r o v o c a n, ~ c o m o ~ e r a ~ e s p e r a b l e, ~ d i f i c u l t a d e s ~}$ similares en los dos grupos; primero, porque esperaban tener que identificar oraciones de una lengua extranjera y segundo, porque todas eran sintetizadas. El hecho más destacable es que confunden en más de un $50 \%$ de los casos el tono [LHH] de la última palabra en las declarativas con el tono de frontera $/ \mathrm{H} \% /$, propio de las interrogativas. No muestran tantos problemas al reconocer las interrogativas sin partícula, ya que, de media, los dos grupos lo hacen mejor que con las interrogativas modificadas de Madrid.

$5^{\text {a) }}$ Teniendo en cuenta los resultados obtenidos y siendo conscientes de la necesidad de análisis futuros estadísticamente probatorios, consideramos que se confirma la primera hipótesis planteada al inicio puesto que los estudiantes japoneses de ELE cometen muchos menos errores en las preguntas del español de Madrid que en las de Tenerife, también encontramos, como se describe en los puntos previos de este mismo apartado, los elementos de la prosodia que más dificultan el reconocimiento del patrón circunflejo y que confunden en mayor medida a los auditores, lo que consideramos que servirá para impulsar mejoras en los procedimientos para la enseñanza de los patrones estudiados y de sus diferencias. De esta forma, creemos que sería de gran importancia entrenar a los estudiantes japoneses de español, así como a otros estudiantes extranjeros, en el reconocimiento de estos dos patrones interrogativos usados ampliamente en el español, enseñando a los alumnos cómo discriminar entre las marcas de acento y modalidad para que puedan percibir y producir correctamente tanto el acento de la última palabra de las oraciones como cualquiera de los dos patrones interrogativos. En caso contrario, estos alumnos se 
verían en graves dificultades comunicativas, como hemos descrito, sobre todo al intentar comunicarse con algún hablante de las variedades que tienen como principal patrón interrogativo el circunflejo.

\section{REFERENCIAS}

Cortés Moreno, M. (2005). "Análisis experimental del aprendizaje de la acentuación y la entonación española por parte de hablantes nativos de chino", in Phonica, 1: 1-25.

Dorta, J. (2008). "La entonación de las interrogativas simples en voz femenina. Zonas urbanas de las Islas Canarias", in A. Turculet (ed.), La variation diathopique de l'intonation dans le domain roumain et roman. Rumanía: Presses de l'Université «Al. I. Cuza» de Iaşi, 123-150.

Dorta, J. (ed.) (2013). Estudio comparativo preliminar de la entonación de Canarias, Cuba y Venezuela. Madrid-Santa Cruz de Tenerife: La Página ediciones S/L, Colección Universidad.

Dorta, J. \& Martín, J.A. (2014). "Estudio preliminar de la fonética y fonología de la entonación de La Habana en el marco de AMPER-Cuba", in Y. Congosto, M.L. Montero and A. Salvador (eds.), Fonética Experimental, Educación Superior e Investigación. T. III. Madrid: Editorial Arco Libros, 189-210.

Dorta, J., Martín, J.A. \& Díaz, C. (2015). “Continuidad prosódica en habla experimental y espontánea de Canarias y Cuba: variación y rango tonal en las interrogativas no pronominales", in K. Kragh and J. Lindschouw (eds.), Les variations diasystématiques et leurs interdépendances dans les langues romanes. Strasbourg: Académie Royale des Sciences et Belles-lettres de Danemark et du Lektor Knud Henders Legatfond, 145-159.

Fernández Lázaro, G. (2009). "Aprendiendo prosodia: la entonación", in Cuadernos CANELA (Confederación Académica Nipona, Española y Latinoamericana), 21: 105-118.

Fernández Lázaro, G., Fernández Alonso, M. \& Kimura, T. (2013). “Corrección de errores de pronunciación para estudiantes japoneses de ELE”, póster presentado en XXV Congreso CANELA (Confederación Académica Nipona, Española y Latinoamericana),Universidad ICU, Mitaka, Tokyo.

Fernández Planas, A.Ma., Dorta, J., Roseano P., Díaz, CH., Elvira García, W., Martín Gómez, J.A. \& Martínez Celdrán, E. (2015). "Distancia y proximidad prosódica entre algunas variedades del español: un estudio dialectométrico a partir de datos acústicos", in $R L A$. Revista de Lingüística Teórica y Aplicada, 53, 2:13-45.

Hara, M. (1990). "Método de enseñanza de la pronunciación española a los alumnos japoneses". Actas del segundo congreso nacional de ASELE, 371-379.

Kimura, T., Sensui, H., Takasawa, M. \& Toyomaru, A. (2008). "El tono HLH* - una señal perceptiva que indica un límite", in Language Design Special Issue, 2: 73-81.

Kimura, T., Sensui, H., Takasawa, M., Toyomaru, A. \& Atria, J.J. (2010). "On Sentence-type Discrimination Strategies of Japanese Learners of Spanish - An Evidence from the HLH* Tone". Poster presented in The Fourth European Conference on Tone and Intonation (TIE4), Universidad de Estocolmo, Campus Frescati.

Kimura, T., Sensui, H., Takasawa, M., Toyomaru, A, \& Atria, J.J. (2012). "Influencia de la entonación española en la percepción del acento por parte de estudiantes japoneses", in Estudios de Fonética Experimental, XXI: 11-42.

Kori, S. (2015). "Nihongo no Bunmatsu Intoneshon no Shurui to Meisho no Saikento [Inventory of sentence-final intonation in Tokyo Japanese: A revisited study]" (en japonés), in Gengo Bunka Kenkyu [Studies in Language and Culture], 41: 85-107. 
Kubozono, H. (1999). "Mora and Syllable", in Natsuko Tsujimura (ed.), The Handbook of Japanese Linguistics. Oxford: Wiley-Blackwell, 31-61.

Martínez Celdrán, E. \& Fernández Planas, A.M. (2003-2015). Atlas Multimèdia de la Prosòdia de l'Espai Romànic. Available from:

http://stel.ub.edu/labfon/amper/cast/index_ampercat.html, accesed 1 Jun, 2016.

Morimoto, Y. (2007). "Algunas aportaciones en torno a los elementos clave de la pronunciación española para los hablantes nativos del japonés", in Bunka Ronshu [The Cultural review] (Waseda Shogaku Dokokai, Universidad Waseda, Facultad de Comercio), 30: 169-187.

Sensui, H. (2014). "Nihonjin Supeingo Gakushusha no Hatsuwa ni Okeru Inritsuteki Tokucho ni Kansuru Keisu Sutadii [A Case Study on Some Prosodic Characteristics of Japanese Students of Spanish]" (en japonés), in Academia (Bungaku Gogaku Hen) [Journal of Nanzan Academic Society ACADEMIA Literature and Language] (Universidad Nanzan), 96: 21-55.

Sensui, H. (2015). Un estudio fonético experimental sobre la percepción de la entonación de oraciones declarativas e interrogativas del español por hablantes nativos y estudiantes japoneses. Ph.D dissertation, Sophia University, Tokyo.

Sensui, H., Kimura, T., Takasawa, M. \& Toyomaru, A. (2008). "Supeingo ni Okeru HLH* Oncho no Kyokai Kakutei Kino ni Kansuru Chikaku Jikken Kenkyu [Un estudio experimental perceptivo sobre la función demarcativa del tono HLH* del español]" (en japonés), in Romansugo Kenkyu [Studia Romanica] (Societas Japonica Studiorum Romanicorum), 41: $52-60$.

Sunden, C. (2013). “Grupu Katsudo o Toriireta Shokyu Supeingo Kyouiku no Kokoromi [Una prueba en una clase elemental de español, usando actividades en grupo]" (en japonés), in Gaikokugo Kyoiku Forum [Forum for Foreign Language Education] (Universidad de Kansai), 12: 147-156.

Takasawa, M., T. Kimura, H. Sensui, A. Toyomaru \& J.J. Atria (2012). "Percepción de la entonación en oraciones compuestas declarativas e interrogativas en español por estudiantes japoneses de español”, in Hispánica (Asociación Japonesa de Hispanistas), 56: 97-112.

Ura, M. (2010). "Gakyshu Iyoku o Takameru Supeingo Jugyo no Ichi Kosatsu [A study of Spanish language class for increasing motivation for learning]" (en japonés), in Bunkyo Daigaku Kokusaibu Kiyo [Journal of the Faculty of International Studies Bunkyo University], 20, (2):17-35.

\section{Agradecimientos}

Este trabajo se ha realizado en el marco del proyecto Estudio comparativo de la entonación y del acento en zonas fronterizas del español (FFI2014-52716-P), proyecto de I+D dirigido por Josefa Dorta del Programa Estatal de Fomento de la Investigación Científica y Técnica de Excelencia, Subprograma Estatal de Generación del Conocimiento. Ministerio de Economía y Competitividad de España.

Agradecemos al profesor Takuya Kimura por su ayuda con los test de percepción en las universidades Sophia y Seisen (Tokio) y a todos los estudiantes que se prestaron a realizar dichos test. Y al profesor Masahiko Mutsukawa por su colaboración en la preparación de las frases del japonés utilizadas en el experimento perceptivo.

Agradecemos al SEGAI la posibilidad de realizar los análisis de datos recogidos en los test de percepción en el Servicio del Laboratorio de Fonética de la Universidad de La Laguna. 